\title{
Produção e caracterização da farinha da casca da banana
}

\author{
Production and characterization of banana peel flour \\ Producción y caracterización de harina de cáscara de plátano
}

\author{
Francyellen Beserra de Oliveira \\ ORCID: https://orcid.org/0000-0002-7368-4293 \\ Universidade Estadual da Paraíba, Brasil \\ E-mail: francyellen.oliveira2014@gmail.com \\ Ângela Maria Santiago \\ ORCID: https://orcid.org/0000-0001-7108-6890 \\ Universidade Estadual da Paraíba, Brasil \\ E-mail: angelamariasantiago01@gmail.com \\ Wanda Izabel Monteiro de Lima Marsiglia \\ ORCID: https://orcid.org/0000-0002-1137-576X \\ Universidade Estadual da Paraíba, Brasil \\ E-mail: wandaequepb@gmail.com \\ Annely Gianni Aragão Barbosa \\ ORCID: https://orcid.org/0000-0003-1238-6054 \\ Universidade Estadual da Paraíba, Brasil \\ E-mail: annely.g1234@gmail.com \\ Maria Celly dos Santos Silva \\ ORCID: https://orcid.org/0000-0001-5844-4083 \\ Universidade Estadual da Paraíba, Brasil \\ E-mail: mariacelly018@gmail.com \\ Amanda Gabriela Moreira Gouveia \\ ORCID: https://orcid.org/0000-0002-1266-8695 \\ Universidade Estadual da Paraíba, Brasil \\ E-mail: amandagouveia59@gmail.com \\ Marina Gonçalves da Silva Nascimento \\ ORCID: https://orcid.org/0000-0001-9053-5719 \\ Universidade Estadual da Paraíba, Brasil \\ E-mail: marigdsn@gmail.com \\ Maciel de Lima Silvestre \\ ORCID: https://orcid.org/0000-0001-7492-401X \\ Universidade Estadual da Paraíba, Brasil \\ E-mail: limamaciel64@gmail.com \\ Pablícia Oliveira Galdino \\ ORCID: https://orcid.org/0000-0001-6996-0550 \\ Universidade Estadual da Paraíba, Brasil \\ E-mail: pabliciaog@hotmail.com \\ Mércia Melo de Almeida Mota \\ ORCID: https://orcid.org/0000-0002-1336-9355 \\ Universidade Federal de Campina Grande, Brasil \\ E-mail:mercia01@gmail.com
}

\begin{abstract}
Resumo
O presente trabalho teve como objetivo a elaboração da farinha da casca da banana (Musa spp.) branqueada por secagem convectiva e determinar os parâmetros físico-químicos e compostos bioativos. As cascas foram lavadas, higienizados com solução de hipoclorito de sódio e branqueadas. Em seguida foram secas em estufa com circulação de ar a $60^{\circ} \mathrm{C}$ e trituradas. Logo após, a farinha foi avaliada quanto aos parâmetros físico-químicos: $\mathrm{pH}$, sólidos solúveis totais $\left({ }^{\circ}\right.$ Brix), relação SST/ATT, acidez total titulável, teor de água, cinzas, sólidos totais e os compostos bioativos: taninos totais (TT) e composto fenólicos totais (CFT). A farinha apresentou um pH de 6,30, superior ao da casca in natura 5,53, o brix de 15,3 e o da casca 6,50 ${ }^{\circ}$ Brix, o teor de água 8,24 (b.u) e da casca 86,2 (b.u). Os CFT encontrados na farinha foram de 124,48 mg EAG/100g, superiores aos encontrados na amostra in natura, 69,44 mg EAG/100g. Os valores dos TT obtidos na farinha foi de, 112,900 mg EAT/100g inferiores ao da casca $394,900 \mathrm{mg}$ EAT/100g. O teor de água da farinha da casca da banana encontra-se dentro dos limites estabelecidos pela ANVISA podendo, portanto, ser armazenado em temperatura ambiente, sem riscos de desenvolvimento de micro-organismos. Os resultados obtidos com relação aos compostos bioativos apontam que a farinha da casca da banana contém valores significativos, podendo ser incorporada na dieta humana.
\end{abstract}

Palavras-chave: Compostos bioativos; Secagem convectiva; Musa spp. 


\begin{abstract}
The present work had as objective the elaboration of banana peel flour (Musa spp.) bleached by convective drying and determine the physicochemical parameters and bioactive compounds. The peels were washed, sanitized with sodium hypochlorite solution and bleached. Then they were dried in an oven with air circulation at $60^{\circ} \mathrm{C}$ and ground. Soon after, the flour was evaluated for physicochemical parameters: $\mathrm{pH}$, total soluble solids ( ${ }^{\circ} \mathrm{Brix}$ ), TSS/ATT ratio, total titratable acidity, water content, ash, total solids and bioactive compounds: total tannins (TT) and total phenolic compounds (CFT). The flour had a pH of 6.30, higher than the in natura peel 5.53, the brix of 15.3 and that of the peel $6.50{ }^{\circ}$ Brix, the water content 8.24 (b.u) and of the peel 86.2 (b.u). The CFT found in the flour was $124.48 \mathrm{mg}$ EAG/100g, which was higher than that found in the in natura sample, $69.44 \mathrm{mg}$ EAG/100g. The TT values obtained in the flour were $112.900 \mathrm{mg}$ EAT/100g lower than in the peel, $394.900 \mathrm{mg}$ EAT/100g. The water content of the banana peel flour is within the limits established by ANVISA and can therefore be stored at room temperature without risk of microorganism development. The results obtained with respect to bioactive compounds indicate that the banana peel flour contains significant values and can be incorporated into the human diet.
\end{abstract}

Keywords: Bioactive compounds; Convective drying; Musa spp.

\title{
Resumen
}

El presente trabajo tuvo como objetivo preparar la harina de cáscara de plátano (Musa spp.) blanqueada por secado convectivo y determinar los parámetros físicoquímicos y los compuestos bioactivos. Las cáscaras se lavaron, se higienizaron con una solución de hipoclorito de sodio y se blanquearon. Luego se secaron en un horno con circulación de aire a $60^{\circ} \mathrm{C}$ y se molieron. Poco después, se evaluaron los parámetros físicoquímicos de la harina: $\mathrm{pH}$, sólidos solubles totales ( ${ }^{\circ}$ Brix), relación SST/ATT, acidez total titulable, contenido de agua, cenizas, sólidos totales y compuestos bioactivos: taninos totales (TT) y compuestos fenólicos totales (CFT). La harina presentó un pH de 6,30, superior al de la cáscara in natura 5,53, el brix de 15,3 y el de la cáscara 6,50 ${ }^{\circ}$ Brix, el contenido de agua 8,24 (b.u) y el de la cáscara 86,2 (b.u). El CFT encontrado en la harina fue de 124,48 mg de EAG/100g, superior al encontrado en la muestra in natura, 69,44 mg de EAG/100g. Los valores de TT obtenidos en la harina fueron 112,900 mg EAT/100g inferiores a los de la cáscara, 394,900 mg EAT/100g. El contenido de agua de la harina de cáscara de plátano está dentro de los límites establecidos por la ANVISA, por lo que puede almacenarse a temperatura ambiente sin riesgo de desarrollo de microorganismos. Los resultados obtenidos con respecto a los compuestos bioactivos indican que la harina de cáscara de plátano contiene valores significativos y puede incorporarse a la dieta humana.

Palabras clave: Compuestos bioactivos; Secado convectivo; Musa spp.

\section{Introdução}

A banana (Musa spp.) destaca-se como uma das frutas mais consumidas mundialmente. Devido ser uma fruta tropical, o clima no Brasil torna-se propício para o seu cultivo em todo o país. Segundo o Instituto Brasileiro de Geografia e Estatística (2019), IBGE, a produção em dezembro de 2019 foi de 7,113 milhões de toneladas de bananas. Atualmente, o Brasil ocupa a quarta posição na produção de banana, tendo a mesma como a segunda fruta mais consumida no país, ficando atrás somente da laranja, com destaque a região Norte e Nordeste (Anuário, 2019).

A casca é caracterizada por ser o principal subproduto da banana, representando cerca de $40 \%$ do peso total da fruta (Castillo-Israel, 2015). Contudo é, geralmente, descartada de maneira inadequada no lixo ou, em alguns casos, como ração animal, causando impactos ambientais. Foi demonstrado que a casca da banana apresenta mais compostos fenólicos que a polpa (Aquino et al., 2016). Além disto, é fonte de fibras, proteínas e minerais (Carvalho, 2015). Segundo Aquino, Salomão, Siqueira, Cecon e Ribeiro (2014) os teores médios de N, P, Fe, Zn e Cu são duas vezes maiores para a casca do que a polpa, já para o $\mathrm{K}$ e $\mathrm{Mn}$, os valores aumentaram para cerca de quatro vezes. A farinha da casca da banana vem sendo utilizada na produção de produtos como: barras de cereais, biscoitos, massas do tipo tagliatelle, massas para rissol, revestimentos comestíveis e cupcakes (Carvalho, 2015; L. M. S. Silva, 2013; Castelo-Branco et al., 2017; Vieira, 2018; Arquelau, 2018; K. V. Silva, 2018).

Estudos tem voltado a atenção para compostos fenólicos, isto em virtude ao efeito da ação antioxidante em patologias, atuando na inibição de radicais livres. Estes por sua vez estão associados ao desenvolvimento de doenças degenerativas como canceres, envelhecimento, doenças cardiovasculares, diabetes, mal de Alzheimer, entre outros (Barros, 2011). Tendo em vista as propriedades nutricionais presente na casca da banana, o seu aproveitamento é essencial tanto para a indústria como a 
incorporação na dieta humana. Assim sendo, este trabalho tem por finalidade a elaboração da farinha da casca da banana branqueada por secagem convectiva e determinar os parâmetros físico-químicos e compostos bioativos.

\section{Metodologia}

Os experimentos foram realizados no Laboratório do Núcleo de Pesquisa e Extensão em Alimentos (NUPEA) e no Laboratório de Química Analítica, ambos do Centro de Ciências e Tecnologia (CCT), no Campus I da Universidade Estadual da Paraíba (UEPB), localizado na cidade de Campina Grande - Paraíba.

\subsection{Matéria prima e preparação da amostra}

As cascas de banana utilizadas, da variedade prata, foram provenientes de frutas selecionadas pelo aspecto aparente, apresentando coloração amarela e sem pontos pretos, obtidas na feira livre da cidade de Campina Grande - PB. Inicialmente, as bananas foram lavadas em água corrente, para a remoção de resíduos agregados a casca, em seguida descascadas e cordadas, com faca inoxidável, no tamanho de $0,5 \mathrm{~cm} \times 3 \mathrm{~cm}$.

\subsection{Branqueamento}

As cascas já cortadas foram imersas na água em ebulição e a vapor, durante os períodos de 2, 3, 4 e 9 minutos, para a inativação da enzima peroxidase, responsável pelo escurecimento enzimático, em seguida foi resfriada em água a temperatura $\pm 10^{\circ} \mathrm{C}$ para evitar a ação prolongada de calor.

\subsection{Caracterização físico-química da casca in natura}

A caracterização físico-química para o produto in natura foram realizadas em triplicata mediante os parâmetros: acidez total titulável (ATT), sólidos solúveis totais ( ${ }^{\circ}$ Brix), teor de água, sólidos totais, razão SST/ATT e cinzas segundo a metodologia descrita por Brasil (2008). O pH foi determinado pelo método potenciométrico.

\subsection{Compostos bioativos da casca in natura}

\subsubsection{Compostos Fenólicos Totais (CFT)}

O extrato produzido para as análises de compostos fenólicos é análogo ao produzido para taninos totais. A metodologia para a determinação dos compostos fenólicos foi realizada utilizando-se o método colorimétrico de Folin Ciocalteu, descrito segundo Watherhouse (2006) com adaptações. No escuro, no tubo de ensaio foi adicionado uma alíquota de $500 \mu \mathrm{L}$ do filtrado (extrato líquido hidroalcóolico), $1625 \mu \mathrm{L}$ de água destilada deionizada e $125 \mu \mathrm{L}$ do reagente de Folin Ciocalteu (RFC) na concentração $2 \mathrm{~N}$, agitado no agitador de tubos vortex por 30 segundos e logo após deixado em repouso por 5 minutos, em seguida adicionou-se $250 \mu \mathrm{L}$ carbonato de sódio (20\%), agitou-se novamente e ficou em repouso por 30 minutos, posteriormente, foi realizada a leitura no comprimento de onda $\lambda=765 \mathrm{~nm}$, no espectrofotômetro UV1800 com duplo feixe da marca SHIMADUZ. A prova em branco foi feita com água destilada, reagente de Folin-Ciocalteau e solução a $20 \%$ de Carbonato de Sódio. O ajuste para curva de calibração com padrão Ácido Gálico foi a partir de soluções com concentrações que variaram de 45 a $225 \mu \mathrm{g} \cdot \mathrm{mL}^{-1}$. Os valores de compostos fenólicos totais foram expressos em mg de equivalente ácido gálico por $100 \mathrm{~g}$ de fruta $\left(\mathrm{mg} \mathrm{AGE} .100 \mathrm{~g}^{-1}\right)$. As leituras foram realizadas em triplicata.

\subsubsection{Taninos Totais (TT)}

O extrato foi obtido segundo Pansera et al., (2003) com adaptações. Foi pesado um grama da amostra e macerado por 1 minuto, em seguida transferido para um balão volumétrico de $25 \mathrm{~mL}$, previamente revestido com papel alumínio, e 
adicionado etanol a 70\%, logo após levado para o banho-maria por 30 minutos. Em ambiente escuro, foi feita a filtração em papel quantitativo Whatman $\mathrm{n} 1$ e transferido para um béquer de $100 \mathrm{~mL}$.

A determinação dos taninos totais foi realizada de acordo com Pansera et al. (2003), com modificações. Foi retirado uma alíquota de $250 \mu \mathrm{L}$ do extrato e transferido para um tubo de ensaio, no qual foi adicionado $2 \mathrm{~mL}$ do reagente de Folin Ciocalteau (RFC) na concentração 1:10 e agitado em agitador vórtex, seguido de um repouso de 5 minutos. Posteriormente, foi adicionado $2 \mathrm{~mL}$ de uma solução aquosa de carbonato de sódio a $8 \%(\mathrm{~m} / \mathrm{v})$, agitado e depois levado para o banho-maria a $40^{\circ} \mathrm{C}$ ao abrigo da luz por 30 minutos. Em seguida foi realizada a leitura no comprimento de onda $\lambda=725 \mathrm{~nm}$ no espectrofotômetro UV-1800 com duplo feixe da marca SHIMADUZ. Para obtenção das curvas analíticas lineares utilizou-se uma solução estoque de ácido tânico PA com concentração de $100 \mathrm{mg} \cdot \mathrm{mL}^{-1}$ e posterior diluição sequencial variando sua concentração de 5 - 100 mg.mL $L^{-1}$, o branco foi utilizado a cada leitura. O conteúdo de taninos totais foi expresso em mg. $\mathrm{L}^{-1}$ de ácido tânico.

\subsection{Preparação da farinha da casca da banana}

As cascas das bananas após serem lavadas, cortadas, branqueadas e pesadas, foram colocadas em bandejas e levadas a uma estufa com circulação de ar forçada na temperatura de $60^{\circ} \mathrm{C}$, até obter massa constante. Esta temperatura foi usada devido ter sido selecionada em trabalhos anteriores com o mesmo resíduo. Após o processo de secagem as cascas foram trituradas e armazenadas em sacos plásticos hermeticamente fechados para evitar a exposição excessiva ao ar. A Figura 1 exibe as etapas de preparação da farinha da casca da banana.

Figura 1. Etapas de preparação da farinha da casca da banana (Musa spp.).

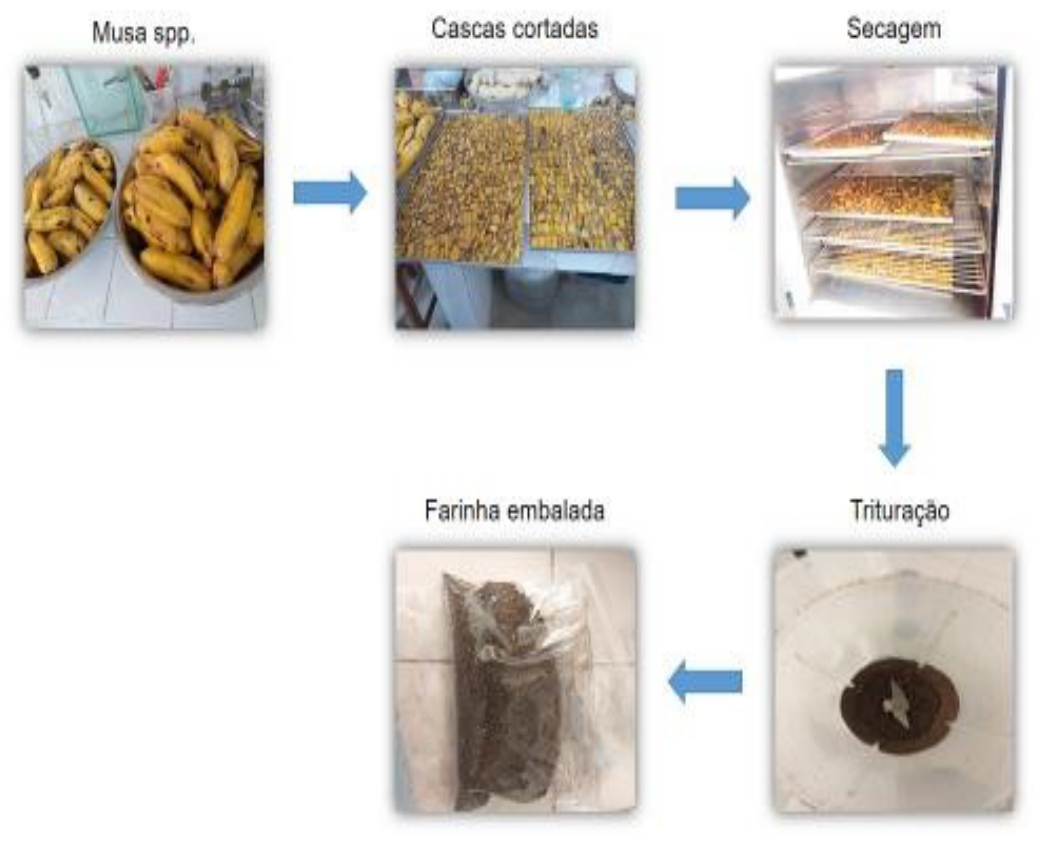

Fonte: Autores (2021).

\subsection{Caracterização físico-química da farinha da casca da banana}

O procedimento físico-químico para a farinha foi determinado mediante os parâmetros: acidez total titulável (ATT), sólidos solúveis totais ( ${ }^{\circ}$ Brix), teor de água, sólidos totais, razão SST/ATT, cinzas e pH, seguindo a metodologia descrita na seção 2.3 . 


\subsection{Compostos bioativos da farinha da casca da banana}

\subsubsection{Compostos Fenólicos Totais (CFT)}

O extrato é idêntico para taninos totais, descrito na seção anterior (2.4.2). A metodologia para a determinação dos compostos fenólicos foi realizada utilizando-se o método colorimétrico de Folin Ciocalteu, descrito segundo Watherhouse (2006) com adaptações. No escuro, no tubo de ensaio foi adicionado uma alíquota de $125 \mu \mathrm{L}$ do filtrado (extrato aquoso), 2 $\mathrm{mL}$ de água destilada deionizada, o restante da metodologia segue conforme descrito para a análise in natura (seção 2.4.1).

\subsubsection{Taninos Totais (TT)}

O extrato foi preparado segundo Pansera et al., (2003) com adaptações. Foi pesado 0,5 da farinha, adicionado $25 \mathrm{~mL}$ de etanol $70 \%$, macerado no almofariz e transferido para um balão volumétrico de $25 \mathrm{~mL}$ previamente revestido de papel alumínio logo após levado para o banho-maria por 30 minutos. Em ambiente escuro, foi feita a filtração em papel quantitativo Whatman $\mathrm{n} 1$ e transferido para um béquer de $100 \mathrm{~mL}$.

A determinação dos taninos totais foi realizada de acordo com Pansera et al. (2003), com modificações. Foi retirado uma alíquota de $250 \mu \mathrm{L}$ do extrato e transferido para um tubo de ensaio, o restante da metodologia segue análogo a determinação de taninos totais para a casca in natura (seção2.4.1).

\section{Resultados e Discussão}

O método de branqueamento selecionado nas cascas de banana foi por imersão em água de ebulição no tempo de 9 minutos, devido ao melhor resultado quando se aplicou o teste qualitativo da presença da enzima peroxidase, que é a mais termoresistente encontrada nos vegetais.

Na Tabela 1 estão apresentados os resultados das análises físico-químicas e dos compostos bioativos para a casca da banana in natura.

Tabela 1. Caracterização físico-química e compostos bioativos presentes na casca in natura.

\begin{tabular}{cc}
$\begin{array}{c}\text { Caracterização Físico-química e Compostos } \\
\text { Bioativos }\end{array}$ & $\begin{array}{c}\text { Média e Desvio } \\
\text { Padrão }\end{array}$ \\
\hline pH & $\mathbf{5 , 5 3} \pm \mathbf{0 , 0 5 8}$ \\
Sólidos Solúveis Totais ( $\left.{ }^{\text {Brix}}\right)$ & $\mathbf{6 , 5 0} \pm \mathbf{0 , 5 0 0}$ \\
Relação SST/ATT & $\mathbf{3 8 , 6 9} \pm \mathbf{0 , 0 0 0}$ \\
Acidez Total Titulável (\%) & $\mathbf{0 , 1 6 8} \pm \mathbf{0 , 0 0 8}$ \\
Teor de água (\% b.u.) & $\mathbf{8 6 , 6 2} \pm \mathbf{0 , 2 9 0}$ \\
Cinzas (\%) & $1,007 \pm 0,425$ \\
Sólidos Totais (\%) & $13,38 \pm 0,290$ \\
Taninos Totais (mg EAT/100g) & $394,90 \pm 0,000$ \\
Compostos Fenólicos Totais (mg EAG/100g) & $\mathbf{6 9 , 4 4} \pm \mathbf{0 , 5 0 0}$ \\
\hline
\end{tabular}

Nota: EAG (Equivalente de Ácido Gálico), EAT (Equivalente de Ácido Tânico).

Fonte: Autores (2021). 
$\mathrm{O}$ pH teve um valor de 5,53 apresentando caráter ácido, similar ao encontrado por Castilho, Alcantara e Clemente (2014), de 5,53, ao analisarem a casca da banana prata em sua pesquisa destinada ao desenvolvimento da farinha utilizando o subproduto da banana maçã e prata. Neris, Silva, Loss, Carvalho e Guedes (2018) ao realizarem a avaliação físico-química da casca da banana (Musa spp.) in natura e desidratada em diferentes estádios de maturação, encontraram um valor próximo, correspondente a 5,84 para a casca da banana maçã madura.

Os sólidos solúveis totais ( ${ }^{\circ}$ Brix) representam os açúcares presentes na fruta, o qual encontramos $6,5^{\circ}$ Brix, idêntico ao valor encontrado por L. M. S. Silva (2013) em seus estudos para o aproveitamento da casca da banana para a produção da farinha destinada a formulação de biscoitos. O mesmo autor encontrou um de teor de água de $84,82 \%$ inferior ao valor encontrado nesta pesquisa que foi de $86,62 \%$, esses resultados elevados de água presente na amostra demonstram que a matéria prima é um produto susceptível a deterioração.

As cinzas correspondem aos resíduos inorgânicos (minerais) presentes na amostra após a incineração da matéria orgânica. Neris et al. (2018) encontraram para o subproduto da banana prata os resultados de cinzas de 1,25 e para a banana do tipo nanica de 1,44, próximos ao valor de 1,007 obtidos neste trabalho. Os resultados de cinzas, analisado por L. M. S. Silva (2013) para a casca in natura foi de 1,68.

Tabela 2. Caracterização físico-química e compostos bioativos presentes na farinha da casca da banana.

\begin{tabular}{cc}
$\begin{array}{c}\text { Caracterização Físico-química e Compostos } \\
\text { Bioativos }\end{array}$ & $\begin{array}{c}\text { Média e Desvio } \\
\text { Padão }\end{array}$ \\
\hline pH & $\mathbf{6 , 3 0} \pm \mathbf{0 , 1 7 3}$ \\
Sólidos Solúveis Totais ( ${ }^{\circ}$ Brix) & $15,30 \pm 0,424$ \\
Relação SST/ATT & $28,49 \pm 0,000$ \\
Acidez Total Titulável (\%) & $\mathbf{0 , 5 3 7} \pm \mathbf{0 , 0 2 7}$ \\
Teor de água (\% b.u.) & $\mathbf{8 , 2 4} \pm \mathbf{0 , 5 8 3}$ \\
Cinzas (\%) & $1,02 \pm 0,015$ \\
Sólidos Totais (\%) & $\mathbf{9 1 , 7 6 \pm 0 , 5 8 3}$ \\
Taninos Totais (mg EAT/100g) & $\mathbf{1 1 2 , 9 0 \pm 0 , 0 0 0}$ \\
Compostos Fenólicos Totais (mg EAG/100g) & $\mathbf{1 2 4 , 4 8 \pm 4 , 4 8 2}$ \\
\hline
\end{tabular}

Nota: EAG (Equivalente de Ácido Gálico), EAT (Equivalente de Ácido Tânico). Fonte: Autores (2021).

A farinha apresentou uma coloração marrom, semelhante a farinha da casca da banana madura da variedade 'Anã', produzida por Fortes et al (2020).

Observa-se que a farinha, assim como a casca in natura, apresentou um comportamento não ácido, com pH 6,30 conforme a classificação dos alimentos quanto ao valor do seu $\mathrm{pH}$ : alimentos ácidos $\mathrm{pH}<4,5$ e alimentos não ácidos $\mathrm{pH}>4,5$. $\mathrm{O}$ pH da farinha analisada por L. M. S. Silva (2013) foi de 6,41 próximo ao encontrado neste trabalho. Silva, Ortiz, Asquiri e Damiani (2020), no entanto, encontraram um pH de 5,84, da mesma forma, Ramos, das Dores Pereira, Andressa, Schmiele e Amaral (2020), obtiveram um resultado de 5,86. 
O teor de água encontra-se dentro dos limites estabelecidos pela ANVISA que é no máximo de $15 \%$ (bu) para farinhas podendo, portanto, ser armazenado em temperatura ambiente, sem riscos de desenvolvimento de fungos.

Em comparação a casca in natura, a farinha da casca da banana apresentou um valor superior de sólidos solúveis totais de $15,30^{\circ}$ Brix. Isto ocorre devido, na farinha, os sólidos estarem mais concentrados, uma vez que há menos água. A farinha produzida por V. D. Silva et al. (2020) e J. S. Silva et al (2020), por sua vez, encontraram um valor inferior ao relatado neste trabalho, de $3,10^{\circ}$ Brix e $6,75^{\circ}$ Brix, respectivamente.

Há poucos estudos associados aos metabólitos secundários presentes na farinha da casca da banana. No entanto, neste estudo, percebemos que os compostos fenólicos totais presentes na farinha 124,48 mg EAG/100 foram superiores aos da casca in natura 69,44 mg EAG/100, isso ocorre porque na farinha os compostos estão mais concentrados, devido a menor quantidade de água disponível. Nos estudos realizados por Pereira (2012), na avaliação da polpa de seis variedades de bananas (figo, terra, maçã, nanica, ouro e prata), os valores de compostos fenólicos variou de 19,90 mg EAG/100g (banana nanica) a 63,94 mg EAG/100g (banana ouro), este último foi próximo ao encontrado neste trabalho para a casca, porém inferiores ao valor da farinha. Isso demonstra a eficácia e valor nutricional acoplado ao aproveitamento do subproduto. Figueredo, Jung, Ribeiro, Kunigami e Nascimento (2019), por sua vez, encontraram uma concentração de CFT de 145 mg EAG/100g, superior ao relatado neste trabalho.

Os compostos fenólicos da farinha avaliada por Arquelau (2018) apresentou um valor de 83,05 mg EAG. 100 $\mathrm{g}^{-1}$, utilizando como método extrativo $\mathrm{MeOH} /$ acetona. Essa diferença nos valores obtidos pode ser explicada pelo método e solvente extrator utilizado. Os valores dos taninos totais na farinha da casca da banana foram de 112,90 mg EAT/100g, inferiores ao encontrado neste trabalho para a amostra in natura, que foi de 394,90 mg EAT/100g, essa diminuição ocorre devido ao aquecimento durante o processo de secagem convectiva.

\section{Conclusão}

O teor de água da farinha da casca da banana encontra-se dentro dos limites estabelecidos pela ANVISA podendo, portanto, ser armazenado em temperatura ambiente, sem riscos de desenvolvimento de micro-organismos.

A farinha da casca da banana produzida neste trabalho apresentou um elevado conteúdo de compostos fenólicos em relação a casca in natura, em detrimento ao processo de secagem que concentra os nutrientes. Entretanto, os conteúdos de taninos totais da amostra seca foram inferiores ao da amostra fresca, devido a exposição ao calor.

Os resultados encontrados nesta pesquisa foram satisfatórios, evidenciando que a farinha da casca da banana contém valores significativos de compostos bioativos, podendo ser incorporada na dieta humana.

\section{Referências}

Alcantara, B. M., Castilho, L. G., \& Clemente, E. (2014). Desenvolvimento e análise físico-química da farinha da casca, da casca in natura e da polpa de banana verde das cultivares maçã e prata. e-xacta, 7(2).

Anuário brasileiro de horti\&fruti 2019. (2018) Santa Cruz do Sul: Editora Gazeta Santa Cruz.

Aquino, C. F., Salomão, L. C. C., Ribeiro, S. M. R., Siqueira, D. L. D., \& Cecon, P. R. (2016). Carbohydrates, phenolic compounds and antioxidant activity in pulp and peel of 15 banana cultivars. Revista Brasileira de Fruticultura, 38(4).

Aquino, C. F., Salomão, L. C. C., Siqueira, D. L., Cecon, P. R., \& Ribeiro, S. M. R. (2014). Teores de minerais em polpas e cascas de frutos de cultivares de bananeira. Revista Agropecuária Brasileira, 49(7).

Arquelau, P. B. F. (2018). Desenvolvimento e caracterização de revestimentos comestíveis a partir de farinha de casca de banana (Musa spp.) Prata (AAB). Dissertação. Universidade Federal de Minas Gerais, Belo Horizonte, Brasil.

Barros, Z. M. P. (2011). Cascas de frutas tropicais como fonte de antioxidantes para enriquecimento de suco pronto. Dissertação de Mestrado. Escola Superior de Agricultura "Luiz de Queiroz", Piracicaba, Brasil. 
Research, Society and Development, v. 10, n. 8, e0910817017, 2021

(CC BY 4.0) | ISSN 2525-3409 | DOI: http://dx.doi.org/10.33448/rsd-v10i8.17017

Castelo-Branco, V. N., Guimarães, J. N., Souza, L., Guedes, M. R., Silva, P. M., Ferrão, L. L., Miyahira, R. F., Guimarães, R. R., Freitas, S. M. L., Reis, M. C. dos., \& Zago, L. (2017). The use of green banana (Musa balbisiana) pulp and peel flour as an ingredient for tagliatelle pasta. Brazilian Journal of Food Technology, 20(0).

Carvalho, V. S. (2015). Aproveitamento da casca da banana na elaboração de barras de cereais: avaliação dos compostos bioativos, características físicas e sensoriais. Tese. Universidade Estadual Paulista “Júlio de Mesquita Filho”, São José do Rio Preto, Brasil.

Castillo-Israel, K. B. (2015). Extraction and characterization of pectin from Saba banana [Musa 'saba'(Musa acuminata x Musa balbisiana)] peel wastes: A preliminary study. International Food Research Journal, 202-207.

Figueiredo, E. S., Jung, E., Ribeiro, L., Kunigami, C., \& Nascimento, F. (2019). Farinha da casca de banana madura: Uma matéria-prima para a indústria alimentícia. Revista Virtual de Química, 11(6).

Fortes, R. R., Brigagão, T. C. S., Lourenço, C. O., Carvalho, E. E. N., Tavano, O. L., Garcia, J. A. D., \& Boas, B. M. V. (2020). Caracterização física e química de farinha de arroz, farinhas de cascas de abacaxi e banana e farinha de sementes de abóbora. Research, Society and Development, 9(9), e436997293e436997293.

Instituto Adolfo Lutz. (2008). Métodos físico-químicos para análise de alimentos. (4ª ed.) (1ª ed. Digital), São Paulo, p.1020.

Instituto Brasileiro de Geografia e Estatística - IBGE. (2019). Levantamento sistemático da produção agrícola: estatística da produção agrícola $<$ https://biblioteca.ibge.gov.br/visualizacao/periodicos/2415/epag 2019 dez.pdf >

Neris, T. S., Silva, S. S. e, Loss, R. A., Carvalho, J. W. P., \& Guedes, S. F. (2018). Avaliação físico-química da casca da banana (musa spp.) In natura e desidratada em diferentes estádios de maturação. Ciência e Sustentabilidade, 4(1), 5-21.

Pansera, M. R., Santos, A. C. A., Paese, K., Wasum, R., Rossato, M., Rota, L. D., Pauletti, G. F., \& Serafini, L. A. (2003). Análise de taninos totais em plantas aromáticas e medicinais cultivadas no Nordeste do Rio Grande do Sul. Revista Brasileira de Farmacognosia, 13(1), 17-22.

Pereira, G. P. (2012). Compostos bioativos e atividade antioxidante em bananas (Musa sp.). Dissertação de Mestrado. Universidade Estadual Paulista "Júlio de Mesquita Filho", São Paulo, Araraquara, Brasil.

Ramos, S. A., das Dores Pereira, R., Andressa, I., Schmiele, M., \& Amaral, T. N. (2020). Desenvolvimento de cookies com coprodutos de frutas. Research, Society and Development, 9(10), e5799108918-e5799108918.

Silva, J. S., Ortiz, D. W., Asquieri, E. R., \& Damiani, C. (2020). Physicochemical and technological evaluation of flours made from fruit co-products for use in food products. Research, Society and Development, 9(3), e192932742-e192932742.

Silva, L. M. S. (2013). Aproveitamento da casca da banana para produção de farinha destinada à formulação de biscoitos. Dissertação de Mestrado. Universidade Federal da Paraíba, João Pessoa, Brasil.

Silva, V. D., Arquelau, P. B., Silva, M. R., Augusti, R., Melo, J. O., \& Fante, C. A. (2020). Use of paper spray-mass spectrometry to determine the chemical profile of ripe banana peel flour and evaluation of its physicochemical and antioxidant properties. Química Nova, 43(5), 579-585.

Silva, K. V. (2018) Elaboração, caracterização sensorial e microbiológica de bolinhos do tipo "cupcake" obtidos a partir da farinha da casca de banana verde. TCC de Bacharel em Nutrição. Universidade Federal de Campina Grande, Paraíba, Cuité, Brasil.

Vieira, B. M. G. (2018). Caracterização de diferentes estados de maturação da farinha da casca da banana e sua aplicação na massa de um rissol sem glúten. Dissertação de Mestrado. Escola Superior de Tecnologia e Gestão do Instituto Politécnico de Viana do Castelo.

Waterhouse, A. L. (2002). Determination of total phenolics. Current protocols in food analytical chemistry, 6(1), I1-1.

Xu, B. J., \& Chang, S. K. C. (2007). A comparative study on phenolic profiles and antioxidant activities of legumes as affected by extraction solvents. Journal of food science, $72(2), \mathrm{S} 159-\mathrm{S} 166$. 